\title{
Silencers are required for inheritance of the repressed state in yeast
}

\author{
Scott G. Holmes and James R. Broach ${ }^{1}$ \\ Department of Molecular Biology, Princeton University, Princeton, New Jersey 08544 USA
}

\begin{abstract}
Transcriptional silencers in the yeast Saccharomyces induce position-specific, sequence-independent repression by promoting formation of a heterochromatin-like structure across sequences adjacent to them. We have examined the role of silencers in maintenance and inheritance of repression at the silent mating-type cassettes in yeast by monitoring the expression state of one of these cassettes following in vivo deletion of the adjacent silencer. Our experiments indicate that although silencer sequences are dispensable for the maintenance of repression in the absence of cell-cycle progression, silencers are required for the stable inheritance of a repressed state. That is, silenced loci from which the silencer is deleted most often become derepressed within one generation of losing the silencer. Thus, the heritability of a repressed state is not intrinsic to a silenced locus or to the chromatin encompassing it; rather, heritability of repression appears to be a property of the silencer itself.
\end{abstract}

[Key Words: Transcriptional silencers; inheritance of repression; S. cerevisiae; mating-type locus; heterochromatin]

Received November 6, 1995; revised version accepted February 27, 1996.

A fundamental property of differentiated cells is their ability to maintain their committed state through many generations, a process that depends on persistence of specific patterns of gene expression from one cell division to the next. One mechanism to ensure this inheritance of gene expression patterns is to have the expression state of a gene promote its own propagation, so that the expression state of a specific locus is a function of its state in the preceding generation. In this strategy, an activating or repressing complex not only acts to regulate the locus but also to template its own reformation during each cell division. Such a mechanism has been proposed to explain the epigenetic pattern of inheritance of heterochromatin-mediated position effects on transcription. For example, female mammals have two copies of the $\mathrm{X}$ chromosome, one of which is maintained in a largely inactive, heterochromatic state. After a single X chromosome is randomly inactivated early in development, the relative expression states of the two $\mathrm{X}$ chromosomes are stable through the life of the organism (for review, see Migeon 1994; Rastan 1994). Both self-templating DNA modification, such as DNA methylation, and self-templating chromatin structure have been proposed to account for this persistence of $\mathrm{X}$-chromosome inactivation (Pfeiffer et al. 1990; Riggs and Pfeiffer 1992).

The yeast Saccharomyces cerevisiae uses a positioneffect mechanism to control the expression of the mating-type genes. Mating type in yeast is determined by genes present at the expressed MAT locus. Identical mat-

${ }^{1}$ Corresponding author. ing-type genes also exist at two other locations, $H M L$ and $H M R$, on the same chromosome. At these sites, however, the mating-type genes are not expressed, even though all the signals for expression are present at the loci (for review, see Herskowitz et al. 1992; Laurenson and Rine 1992). This position-dependent, gene-independent repression, known as silencing, extends to other yeast genes placed at $H M L$ and $H M R$ and results from formation of a specific chromatin structure that appears to be the yeast equivalent of metazoan heterochromatin (Thompson et al. 1993; M. Braunstein, C.D. Allis, B.M. Turner, and J.R. Broach, in prep.).

Elements essential for repression at $H M L$ and $H M R$ have been defined genetically. Silencing depends on the concerted action of cis-acting sequences that flank $H M L$ and $H M R$, known as the $\mathrm{E}$ and I silencers (Abraham et al. 1984; Feldman et al. 1984; Brand et al. 1985; Mahoney and Broach 1989|, and several trans-acting factors, including the products of the SIR genes (Haber and George 1979; Klar et al. 1979; Rine et al. 1979; Rine and Herskowitz 1987). The $E$ and I silencers are small $|<250 \mathrm{bp}|$ regions that confer $S I R$-dependent silencing on genes placed adjacent to them. Silencers consist of specific combinations of two or more binding sites for any of three DNA-binding proteins-Rapl, Abfl, and ORCand silencer activity requires binding of these proteins to their sites (Kurtz and Shore 1991; Bell et al. 1993; Fox et al. 1995; Loo et al. 1995a,b). Deletion of the E site from $H M R$ relieves repression of the locus, whereas both $\mathrm{E}$ and I must be deleted to induce expression at HML. The functions of the products of the SIR genes are not 
Figure 1. Elimination of the $H M L$-E silencer by in vivo recombination. (Top) Diagram of the HML locus in strain Y2048. In this strain, the HML locus (encompassing the homology regions $\mathrm{W}, \mathrm{X}$, and $\mathrm{Z}$, and the repressed genes $\alpha 1$ and $\alpha 2)$ carries a deletion of the I silencer on the centromere-proximal side and an intact $\mathrm{E}$ silencer on the centromeredistal side. A FRT site /shaded box; arrow below indicates the orientation of the site) lies between $E$ and the silenced genes but does not affect the repression of $\alpha 1$ and $\alpha 2$ by E. Centromere distal to the intact $E$ site is an E-site deletion adjacent to a second $F R T$ site in direct orientation with the first site. Induction by galactose of the GAL1O-FLP1 gene integrated elsewhere in the genome results in the diagramed recombination event to yield the locus configuration shown at the bottom, in which the HML locus is now bracketed by deletions of both $\mathrm{E}$ and $\mathrm{I}$.

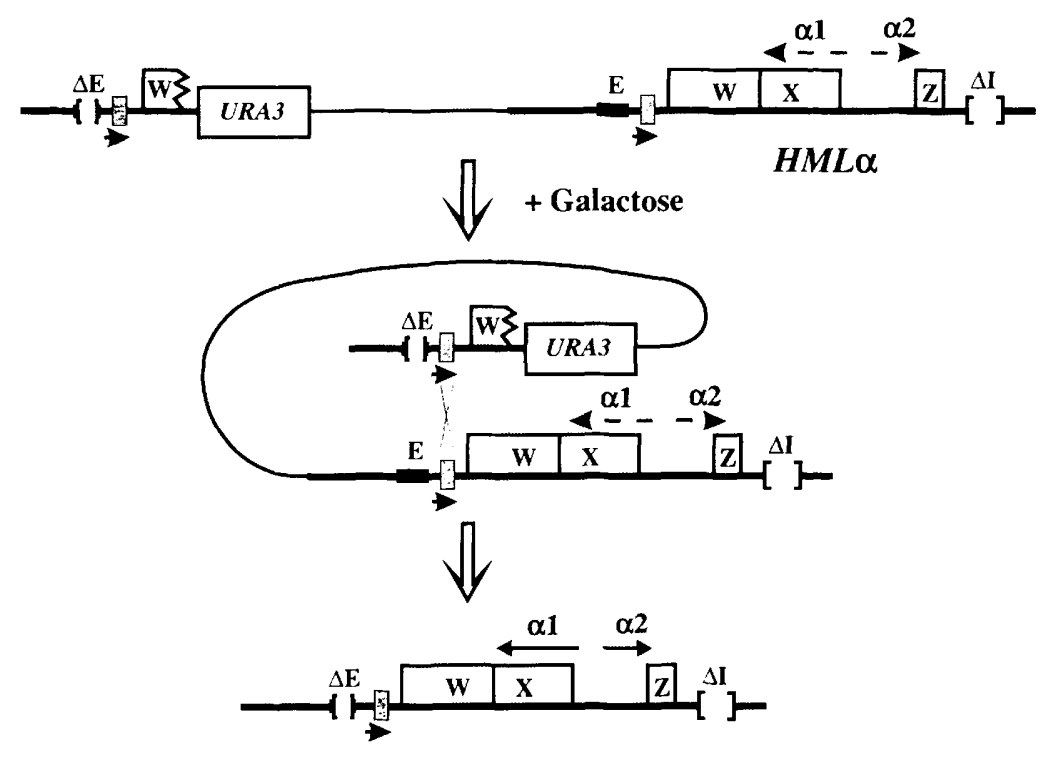

known. Because Sir3p and Sir4p can bind to histones H3 and $\mathrm{H} 4$ (Hecht et al. 1995) and Sir2p affects the acetylation state of nucleosomes in silenced chromatin (Braunstein et al. 1993|, however, these genes may participate directly in determining the specific chromatin structure over the silent loci. Moreover, null mutations in the SIR2, SIR3, or SIR4 genes lead to a complete loss of repression (Rine and Herskowitz 1987).

Certain mutations that attenuate, but do not eliminate, silencing have revealed an epigenetic mode of inheritance of the repressed state of the silent cassettes. Null mutations in SIR1 (Pillus and Rine 1989) and certain mutations in the HML E silencer (Mahoney et al. 1991) or in RAP1 (Sussel et al. 1993) yield a mixed population of completely repressed and completely derepressed cells. These cells exhibit a stable inheritance of their expression state; repressed cells gave rise to repressed cells at a far higher rate than would be predicted from their ratio in the population, and vice versa. This observation suggests that the repressed state is self-templating; once established, the repressive structure can enhance the probability that it will be inherited in the next generation. In addition, these observations suggest that silencing can be divided into two stages: an establishment phase and a maintenance phase (Pillus and Rine 1989; Sussel et al. 1993|. In this view, Sirlp and the silencer sequences play a primary role in establishing repression. Once the repressive structure is established, Sirlp and the silencers would not be required for the subsequent maintenance of repression.

We have examined the mechanism underlying the epigenetic inheritance of silencing by defining the role of silencer sequences in the maintenance and inheritance of the repressed state. We have constructed a strain in which the silencer sequences could be deleted by an inducible, in vivo recombination event and then examined the fate of the adjacent silent cassette following deletion of the silencer. Our experiments indicate that the heri- tability of the transcription state is not intrinsic to the silenced locus or the chromatin encompassing it; rather, it appears to be a property of the silencer. These results suggest that heterochromatin is not intrinsically selftemplating but that epigenetic inheritance observed in many cases of position-effect repression may result from specific sequences or mechanisms that exist to ensure stable persistence of repression.

\section{Results}

HML is readily accessible to FLP1 recombinase

To investigate the role of the $H M L$ silencers in the maintenance and inheritance of transcriptional repression, we constructed strain Y2048 in which the silencer could be deleted by an inducible in vivo recombination event (see Fig. 11. HML is flanked by two silencers, E and I, either of which alone is sufficient for silencing the locus $(\mathrm{Ma}-$ honey and Broach 1989l. Accordingly, we started with a strain in which the I silencer had been deleted and then introduced $F L P 1$ recombination target $(F R T)$ (Broach and Volkert 1991/) sites in direct orientation on either side of the E silencer. In this fashion, repression of $H M L$ could be maintained as long as FLP1 recombinase was not expressed the presence of the FRT sites flanking the $\mathrm{E}$ silencer in this strain did not diminish the ability of the silencer to repress expression from $H M L$, as judged by quantitative mating (data not shown) and $\alpha$-factor confrontation assays (see below)]. At the desired time, Flp1ppromoted recombination between the two FRT sites could delete the $\mathrm{E}$ silencer to yield a configuration that does not support silencing of $H M L$. The configuration resulting from $F L P$-mediated excision consists of the J79-119 deletion allele of $E$ adjacent to $W$ with the 80-bp $F R T$ site lying between them (Fig. 1). The E deletion allele lacks the RAP1 and autonomously replicating sequence (ARS) elements that comprise the E silencer and 
retains no measurable silencing activity (Mahoney and Broach 1989; see below). Conditional expression of FLP1 was obtained from a single, integrated copy of GAL10 FLP1, which was inactive when cells were grown in the absence of galactose but rapidly inducible following transfer to a galactose-containing medium. Thus, growth of this strain in the absence of galactose should retain the silencer at $H M L$; transfer to galactose should lead to rapid excision of the silencer from $H M L$.

Because $H M L$ and $H M R$ have been shown previously to exhibit limited accessibility to a variety of proteins, including DNA repair factors (Terleth et al. 1989), DNA methylases (Singh and Klar 1992), and restriction endonucleases (Loo and Rine 1994), we first examined whether Flplp could promote rapid and efficient excision of the $\mathrm{E}$ silencer from $H M L$ in our strain. Strain Y2048 was grown to $10^{7}$ cells $/ \mathrm{ml}$ in YP-raffinose, a medium that neither induces nor represses the GAL10 promoter. The culture was then split, and galactose was added to one of the parallel cultures. Cells were removed from both cultures at various times following induction, and the extent of excision of the $\mathrm{E}$ silencer was determined by both a physical and a genetic assay. In the physical assay, the proportion of deleted genomes to nondeleted genomes was calculated by quantitation of Southern blots of genomic DNA isolated from the culture samples and probed with a fragment spanning the $\mathrm{E}$ region. In the second assay, cells were plated on a glucose-containing medium, which represses expression of FLP1, and, because Flplp has a very short half-life in vivo (Volkert and Broach 1986), precludes further Flplp-mediated recombination events. Accordingly, those cells that retained the E site at the time of sampling would yield a colony in which most cells maintained $H M L$ in its repressed state and thus were capable of mating. Those cells that had lost the E site at the time of sampling would yield colonies in which all of the cells were derepressed at $H M L$ and thus were incapable of mating. Therefore, the proportion of nonmating to mating colonies represents the proportion of cells in which the $E$ site had been deleted at the time of sampling. As shown in Figure 2, both assays gave identical results and demonstrated that $>90 \%$ of the cells undergo silencer deletion within $30 \mathrm{~min}$ of induction, that is, within less than a single generation. We conclude from these results that FLP1-mediated deletion of the E silencer in this strain is both efficient and readily controlled. In addition, the genetic assay confirms that deletion of the $E$ site in this strain ultimately results in full expression of $H M L$.

\section{Silencers are not continuously required for maintenance of repression}

To determine the immediate effects of deletion of the silencer on expression of $H M L$, we used sensitivity to mating pheromone as an assay for expression of $H M L$. The mating pheromone $\alpha$-factor arrests haploid a cells in the $\mathrm{G}_{1}$ phase of the cell-division cycle but does not affect cell-cycle progression of cells expressing both $a$ and $\alpha$ mating-type information. Therefore, an a strain in which

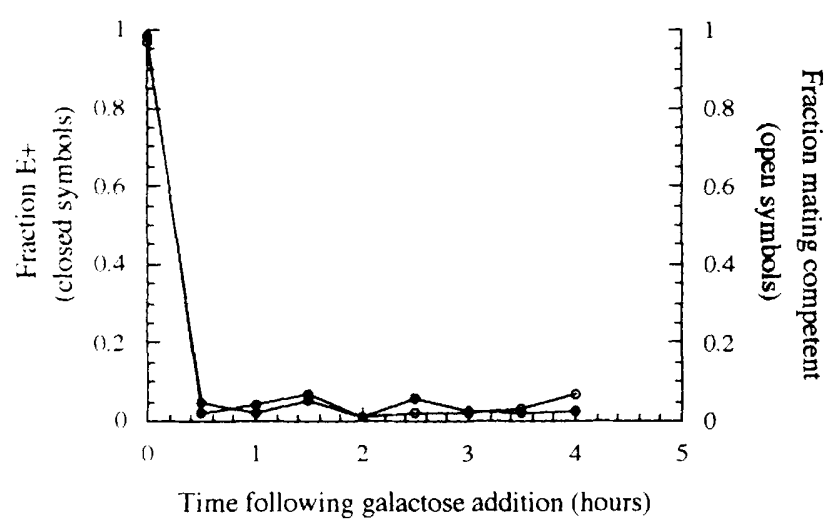

Figure 2. $H M L$ is accessible to Flplp recombinase. Strain Y2048 was grown to $10^{7}$ cells $/ \mathrm{ml}$ in YP-raffinose, and galactose was added to $2 \%$ at time $=0$. The percentage of cells retaining $H M L E$ at the indicated times following induction was determined as described in Materials and methods both by Southern blot analysis $(O)$ and by mating assays $|O|$. Several independent experiments were performed with similar results; a representative experiment is shown. In all trials, $<1 \%$ of cells in a parallel uninduced culture had deleted $\mathrm{E}$.

$H M L \alpha$ is completely silenced is fully sensitive to $\alpha$-factor, whereas an a strain that has lost silencing of $H M L \alpha$ expresses both a and $\alpha$ information and therefore is resistant to arrest by $\alpha$-factor (Pillus and Rine 1989; Mahoney et al. 19911.

The design of this experiment is diagramed in Fig. 3A. We added $\alpha$-factor to strain Y2048 growing exponentially in YP-raffinose medium. Greater than $90 \%$ of the cells in the culture arrested growth within a single generation to produce a near uniform population of unbudded cells, consistent with $H M L \alpha$ in this strain being efficiently repressed. After cell-cycle arrest, we split the culture in two. We maintained one portion as an uninduced culture in raffinose medium and added galactose to the other portion to induce deletion of the silencer. If deletion of the silencer resulted in the immediate expression of $H M L \alpha$, then cells would have become resistant to $\alpha$-factor and resumed progression through the cell cycle. This would have been evident by a decrease in the number of unbudded cells. If silencing persisted in the absence of the silencer, however, then the cells would have remained sensitive to $\alpha$-factor and the number of unbudded cells would not have decreased.

The results of this experiment are shown in Figure 3B. Induction of FLP1 gave very efficient excision of the silencer under these conditions; $>90 \%$ of the cells in the induced culture lost the silencer within $30 \mathrm{~min}$ of induction. Despite the fact that the silencer was deleted from most of the cells in the population, the percentage of unbudded cells in the induced culture remained identical to that in the uninduced culture or to that of a parallel culture of the parent strain in which the $E$ site was not bracketed by $F R T$ sites. All three cultures remained arrested in $G_{1}$ for $\sim 5 \mathrm{hr}_{\text {; }}$ after this time, cells resumed budding in all three cultures as a result of adaptation to 


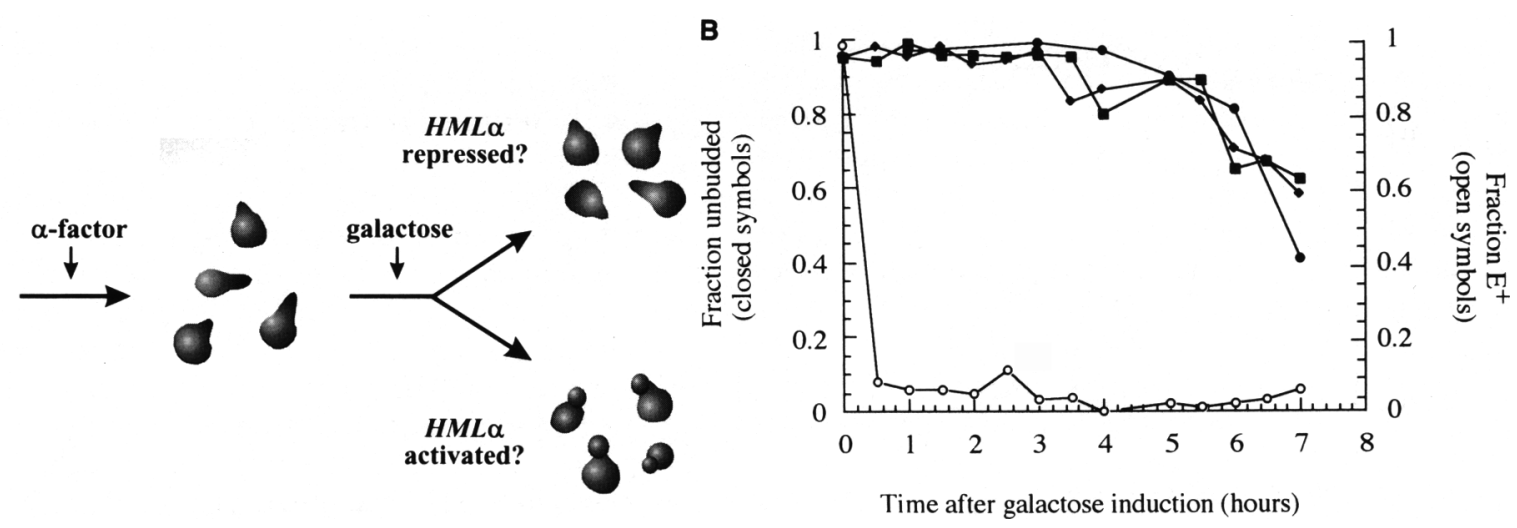

Figure 3. The silencer is not required for maintenance of $H M L$ repression in cells arrested in $\mathrm{G}_{1}$. $(A) \alpha$-Factor sensitivity assay for $H M L \alpha$ expression. To a growing culture of the test strain $(l e f t), \alpha$-factor is added to induce cell cycle arrest at $\mathrm{G}_{1}$ and a concomitant unbudded and anisotropic morphology (middle). Addition of galactose to the arrested cells results in deletion of the $\mathrm{E}$ silencer; Southern blotting was used to confirm that the deletion occurred while the cells were at the $G_{1}$ block. The effect of the deletion on expression of $H M L$ can be assessed by whether the cells remain unbudded ( $H M L$ is repressed) or resume budding ( $H M L$ is activated). (B) The indicated strains were grown to $\sim 10^{7}$ cells $/ \mathrm{ml}$ and treated with $\alpha$-factor to induce uniform arrest. Galactose was then added at time $=0$ to cultures of $Y 2048$ and the control strain Y728. At the indicated time the fraction of Y2048 cells retaining $H M L E(O)$ was determined as described in Material and methods, and the fraction of unbudded cells was determined by microscopic examination of the induced Y2048 (-) and Y728 (O) cultures as well as of a parallel uninduced culture of $\alpha$-factor arrested Y2048 cells ( $\$$ ). A representative experiment is shown.

the mating pheromone. Thus, these results provide evidence that for cells arrested at $G_{1}$, the silencer element is not required for maintenance of repression at the silent mating-type locus.

An alternative explanation for these results is that $H M L \alpha$ is immediately derepressed following deletion of the silencer but that derepression does not lead to immediate resistance to $\alpha$-factor. To address this question of phenotypic lag, we integrated a plasmid containing the $\alpha 2$ gene controlled by a GAL10 promoter into our strain background and performed an experiment similar to the one described above (see Fig. 4). The promoter used in these studies was attenuated such that fully induced $\alpha 2$ expression was not substantially higher than that obtained from the normal $\alpha 2$ gene (Mahoney et al. 1991). Cells were grown in YP-raffinose, arrested with $\alpha$-factor, and then induced with galactose. In this case, induction of the $\alpha 2$ gene resulted in a rapid release from cell-cycle arrest. This demonstrates that if deletion of the silencer at $H M L \alpha$ had resulted in activation of the locus, then Y2048 cells would have escaped $\alpha$-factor arrest following induction of FLP1. From these experiments, we can conclude that the cis-acting silencer sequences are not continuously required to maintain transcriptional repression.

\section{Sir $3 p$ is required for silencing at a $G_{1}$ block}

The results described above for deletion of the silencer from $H M L$ are at odds with those obtained by Miller and Nasmyth (1984), who showed that inactivation of SIR3 in cells arrested at $G_{1}$ leads to immediate activation of the silent cassettes. The difference in the two outcomes could be a trivial result of the different assay procedures, or it could reflect a fundamental distinction between the two modes of $H M L$ activation. To distinguish between these two possibilities, we investigated the requirement for SIR3 in maintenance of $H M L$ repression in $\mathrm{G}_{1}$-arrested cells in our assay and strains. We introduced the temperature-sensitive sir3-8 allele (Rine and Herskowitz 1987) into our strain background to create strains Y2049. We grew strain Y2049 in YP-raffinose at $23^{\circ} \mathrm{C}$, at which sir $3-8$ is fully active, and then arrested cells with $\alpha$-factor. The culture was divided in two, and one of the par-

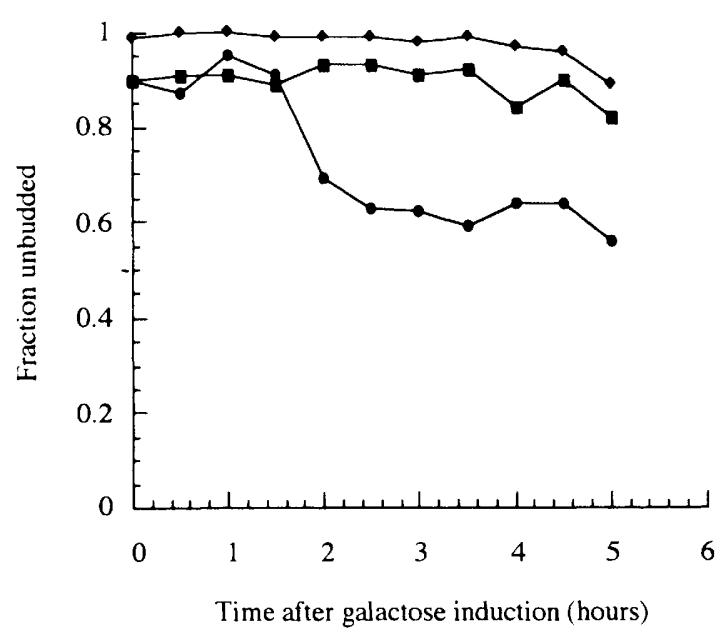

Figure 4. $\alpha 2$ expression results in escape from an $\alpha$-factor arrest. Strain Y1289 $(\mathbf{O})$, which carries the $\alpha 2$ gene under control of the GAL10 promoter, and the control strain Y728 (a) were grown and treated as described in the legend to Fig. 3 . The fraction unbudded cells are shown as a function time following induction with galactose. The budding index of a parallel uninduced culture of Strain Y1289 ( $)$ is also shown. 


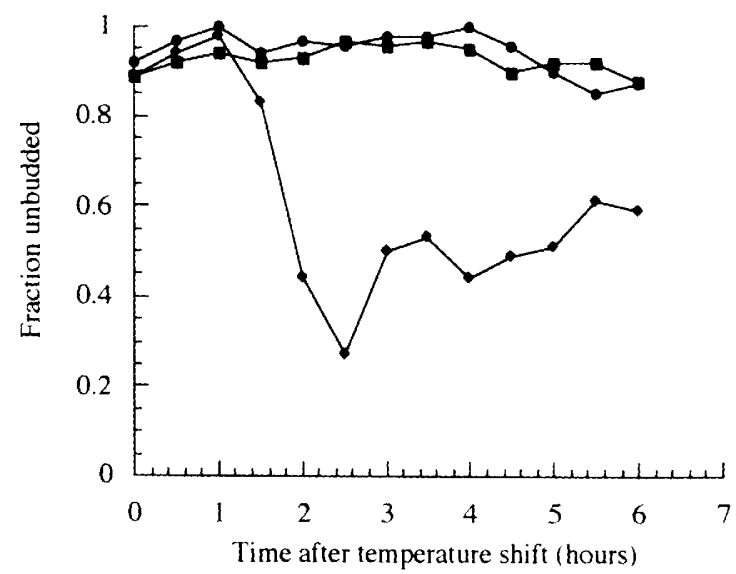

Figure 5. Sir3p is required for maintenance of $H M L$ repression in cells arrested at $G_{1}$. Strain Y2049, carrying a temperature sensitive allele of the SIR3 gene, was grown to $\log$ phase $110^{7}$ cells $/ \mathrm{ml}$ ) at $23^{\circ} \mathrm{C}$ in $\mathrm{YP}$-raffinose. At time $=0$ the culture was divided and grown at $23^{\circ} \mathrm{C}(\mathbf{D})$ or $37^{\circ} \mathrm{C}(1)$. At the indicated times, the fraction of unbudded cells was determined by microscopic determination of each culture. Budding morphology was also determined for control strain $\mathrm{Y} 728$, which had been grown at $23^{\circ} \mathrm{C}$ and shifted to $37^{\circ} \mathrm{C}|\mathbf{O}|$.

allel cultures was maintained at the permissive temperature while the other was shifted to $37^{\circ} \mathrm{C}$ to inactivate sir3-8. As shown in Figure 5, we found that sir3.8 cells incubated at the permissive temperature or SIR 3 cells incubated at either temperature remained arrested during the course of the experiment. In contrast, sir3-8 cells incubated at the elevated temperature escaped $\alpha$-factor arrest with kinetics similar to that of galactose-induced strain Y1289, which carries the GAL10- $\alpha 2$ gene. Thus, we find that, as reported by Miller and Nasmyth (1984), loss of SIR3 function led immediately to expression of $H M L \alpha$. Because this was not the case following deletion of the silencer, we conclude that silencers participate in transcriptional repression of the silent cassettes in a manner quite distinct from that of SIR3.

\section{Silencers are required for inheritance of the repressed state}

Our observation that silencers are not continuously required to maintain repression is consistent with a model in which the silencer sequences function primarily to establish the repressive structure but do not participate in the subsequent maintenance or inheritance of the structure. To test this hypothesis, we examined the role of silencers in the inheritance of the repressed state by performing pedigree analysis on cells that had undergone the FLP-mediated silencer deletion, as outlined in Figure 6. As before, cells were grown in YP-raffinose, arrested with $\alpha$-factor, and then induced with galactose. After allowing sufficient time for deletion of the silencer, cells were transferred to a glucose-containing plate adjacent to a source of $\alpha$-factor. Cells still arrested in $G_{1}$ were identified microscopically and manipulated away from the source of $\alpha$-factor to allow them to resume progress through the cell cycle. When these cells budded, they were returned to the presence of $\alpha$-factor. Following cell division, the mother and daughter cells were separated and assayed for their response to $\alpha$-factor. By this procedure we could determine the expression state of $H M L$ exactly one generation after the deletion of the silencers.

As depicted in Figure 6, we could envision three different responses for cells subjected to this regime. If repression of $H M L$ were stable in the absence of the silencers, then both the mother and daughter cell would remain sensitive to $\alpha$-factor, even though the silencer had been deleted in the previous generation. Thus, both cells would fail to grow when challenged with $\alpha$-factor. On the other hand, if the repression state were not inherited in the absence of the silencer, then $H M L$ would be expressed in both the mother and daughter and both would continue growing in the presence of $\alpha$-factor and form microcolonies. Finally, if the effect on repression of the deletion of the silencer were not absolute, then we might anticipate that some cells would yield mothers and daughters that behaved differently when challenged with $\alpha$-factor, with one arresting and the other growing.

The results of our pedigree analysis are shown in Table 1. When this experiment was performed with a culture that was not induced with galactose, cells most often gave rise to progeny that arrested in the next generation. This was expected, because the progeny all retained the silencer and thus remained repressed at $H M L$ (the unusual classes are discussed belowl. In contrast, cells from the culture that was induced to delete the silencers most often gave rise to progeny that were resistant to $\alpha$-factor in the next generation. We conclude from this experiment that even though silencers are not required for maintaining repression in arrested cells, they are re-

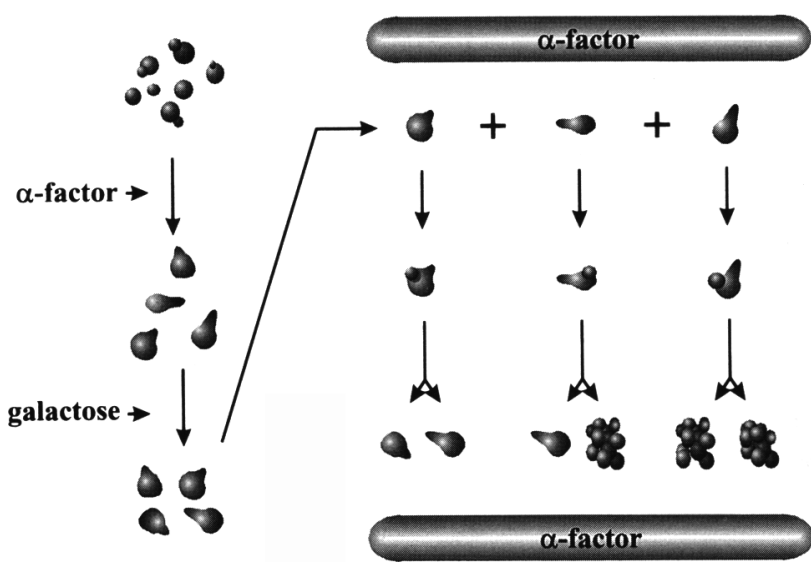

Figure 6. Pedigree analysis of cells lacking silencers. Strain Y 1289 was grown to $\log$ phase, then arrested in $G_{1}$ with $\alpha$-factor, when galactose was added to induce the silencer deletion. Cells were then transferred to a plate containing a source of $\alpha$-factor. Individual cells were moved away from the influence of pheromone and allowed to bud, when they were returned to $\alpha$-factor. Following cytokinesis, cells were separated and cell pairs were then assayed for their response to pheromone. 
Table 1. Instability of repression in the absence of silencer sequences

\begin{tabular}{lcccc}
\hline & \multicolumn{3}{c}{ Fraction of cell pairs } \\
\cline { 2 - 5 } Pregrowth & & & & \\
condition & + & 0.10 & 0.84 & 192 \\
\hline+ galactose & 0.06 & 0.08 & 0.09 & 127 \\
\hline
\end{tabular}

quired for efficient inheritance of the repressed state. Thus, the repression complex over the silent cassette that is sufficient to maintain repression is not sufficient to promote its propagation from one generation to the next.

The repressed state can be inherited rarely in the absence of the silencer

Examination of the unusual classes of cells in the pedigree analysis revealed that in a few instances, the repressed state of the silent cassette could be inherited even in the absence of the silencer. The unusual classes included (1) both progeny of individual cells from the induced culture becoming arrested, (2) both progeny of individual cells from the uninduced culture giving rise to microcolonies, and $(3)$ the two progeny of individual cells from either culture giving different responses. As discussed below, the first two classes arise from technical limitations of the experiment. Some members of the third class, however, arise from silencer-independent inheritance of repression.

The cells from the induced culture that gave rise to two progeny that arrested were those cells in which the silencer had not been excised during the period of induction. This was shown by Southern analysis of genomic DNA from cultures obtained from these progeny cells, after removing them from the presence of $\alpha$-factor. The frequency with which they arose in the pedigree, $\sim 7 \%$, was in good agreement with the proportion of undeleted cells present following induction of the test strain, as judged by Southern analysis of DNA from the total culture (cf. Figs. 2 and 3).

Those cases in which progeny from cells from the uninduced culture grew in the presence of $\alpha$-factor, regardless of whether both or only one of the progeny grew, were apparently attributable to adaptation of these cells to $\alpha$-factor during the course of the experiment (Reed 1991). All of these microcolonies retained the E silencer, as judged by Southern analysis of cultures obtained from them and by the fact that within several generations all of the cells in the microcolonies arrested growth. We confirmed this hypothesis by showing that neither of these unusual classes arose when we performed the same experiment on a strain in which we had introduced an sst2 mutation, which prevents adaptation to $\alpha$-factor exposure. Thus, we conclude that the spontaneous deletion rate of the silencer in uninduced cultures is $<1 \%$ per generation, but that under the conditions of the experiment, $\sim 10 \%$ of the sensitive cells can adapt to $\alpha$-factor resistance.

Finally, most of the cases in which the two progeny of individual cells from the induced culture responded differently to $\alpha$-factor cannot be explained by either of these events. In 14 of 16 pairs of such cells examined, both of them appear to have been deleted from the silencer at the time of $\alpha$-factor confrontation. In these cases, the resistant clone of each pair remained resistant during extended growth in the presence of $\alpha$-factor, and Southern analysis of the resulting clonal culture indicated that the $E$ site had been deleted. The sensitive sister to these cells gave rise to two resistant progeny when removed from $\alpha$-factor, allowed to undergo a single round of cell division, and then returned to the presence of $\alpha$-factor. Southern analysis of clonal cultures of these progeny documented the absence of the $\mathrm{E}$ site in all cells. This indicates that this class is not the result of a FLP event that occurred in one cell after DNA replication. In that case, the two cells would give rise to one colony with an intact silencer and one with a deleted silencer. This was not observed. Thus, we conclude that the majority of the members of this class began as cells from which the $\mathrm{E}$ site had been deleted during the induction period. Whereas in one of the progeny the HML locus was activated during cell division, however, the other progeny retained $H M L$ in its repressed state through the single round of the cell cycle. From a limited sampling, no mother-daughter bias was evident as to which of the two cells remained silenced. Therefore, we conclude that at a very low frequency, the repression state of $H M L$ can persist through a complete cell cycle even in the absence of a silencer. From our data, the probability of inheriting the repressed state in the absence of a silencer is $\sim 5 \%$.

\section{Discussion}

Deletion and reconstruction studies have demonstrated previously that silencer sequences are necessary and sufficient to mediate transcriptional repression; removal of the silencer sequences leads to full expression of the silent mating-type loci (Brand et al. 1985; Mahoney and Broach 1989), whereas introduction of silencer sequences adjacent to the expressed MAT locus renders it transcriptionally inactive (Shei and Broach 1995). We have defined the precise functions of the silencers by using site-specific recombination to delete the sequences in vivo. We find that maintenance of the repressive structure at $H M L$ does not depend on the presence of the silencer; in $G_{1}$-arrested cells repression is unaffected when the sequences are deleted. These results are consistent with an in vitro study demonstrating that $H M R$ remained inaccessible to restriction endonucleases when separated from its silencers (Loo and Rine 1994). We find, however, that repression in the absence of the silencers is erased in cycling cells within a single generation. This indicates that silencers are required for inheritance of the repressed state. 


\section{Heterochromatin managers and workers}

Our results, which are summarized in Figure 7, establish a marked distinction in the roles of different components of the silencing apparatus. Inactivation of SIR3 in cells arrested at $G_{1}$ results in immediate loss of repression of the silent locus, whereas deletion of the silencer has no effect on repression as long as cells remain cell-cycle arrested. The effect of deletion of the silencer becomes manifest only when the locus is subjected to the various manipulations imposed by the cell cycle. Our interpretation of these results is that Sir3p participates directly as an essential component of the heterochromatin spanning the silent loci. Accordingly, when it is inactivated, the heterochromatic structure immediately disintegrates. In contrast, our results argue that the silencer complex acts to position heterochromatin and promote its persistence from one generation to the next, but it does not comprise an integral component of the heterochromatin. Accordingly, even when it is eliminated, the heterochromatin remains intact until being disrupted by cell-cycle events. An alternate hypothesis, which is not ruled out by our experiments, is that in $G_{1}$-blocked cells, the induced recombination event uncouples the silencer sequences from $H M L \alpha$, but the silencer continues to exert an influence on the transcription of $H M L \alpha$, possibly through protein-protein interactions. Such a trans-effect has not been described in yeast and we consider this a less likely possibility.

Besides Sir3p, at least two other proteins-Sir $2 p$ and Sir4p-likely fall into the category of integral heterochromatin components. While the kinetics of silent cassette activation resulting from inactivation of SIR2 or $S I R 4$ have not been determined, other studies place these

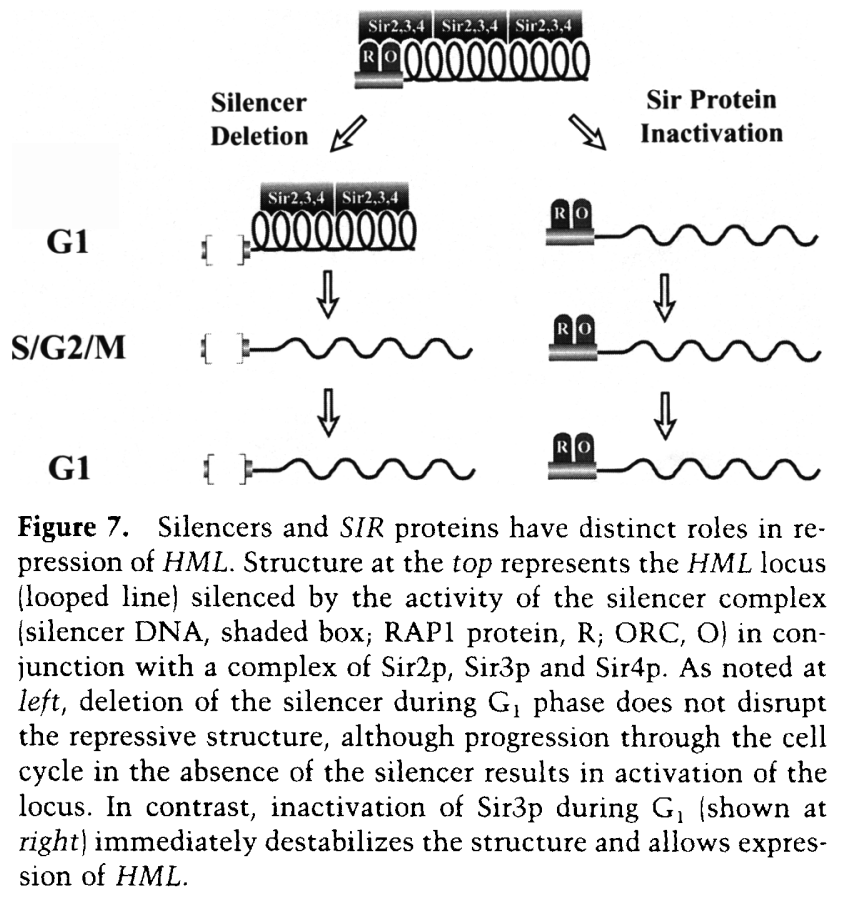

two genes in the same functional group as SIR3. Sir3p and Sir $4 \mathrm{p}$ both show binding to the histone $\mathrm{H} 4$ aminoterminal domain (Hecht et al. 1995), SIR3 overexpression suppresses phenotypes associated with SIR4 overexpression (Marshall et al. 1987), and mutations of SIR3 and SIR4 show similar affects on nuclear localization of Rapl protein (Palladino et al. 1993). Similarly, SIR2 and $S I R 3$ each exhibit overexpression lethality dependent on the integrity of the other (A. Rose and J.R. Broach, unpubl.) and Sir $2 \mathrm{p}$ and Sir3p can be coimmunoprecipitated (E. Saez and J.R. Broach, unpubl.). Thus, all three proteins are likely to be involved in the same process, which we suggest is participating as a structural component of yeast heterochromatin.

In addition to the silencer itself, silencer-binding proteins-Raplp, Abflp, and ORC-as well as Sirlp, likely belong in the category of silencing components that serve to organize silencing but do not participate directly in heterochromatin structure. Most of these components have not been tested in a kinetics of activation assay, but RAPl, ORC, ABF1, and Sirlp all function through the silencer [although Sirlp does not bind directly to the silencer, silencer activity can be partially reconstituted by tethering Sirlp to DNA at the site of a deleted silencer [Chien et al. 1993)]. In addition, mutation of several of these components yields a common silencing phenotype: a partial silencing defect that exhibits epigenetic inheritance. That is, hypomorphic mutations of the HM6L E silencer (Mahoney et al. 1991), certain mutations of RAP1 (Sussel et al. 1993), and null alleles of SIR1 (Pillus and Rine 1989) yield mixed populations of repressed and derepressed cells, each of which gives rise at high frequency to progeny with the same expression state as the parents. This common phenotype suggests that all three elements play similar roles in silencing. Sufficient alleles of $O R C$ and $A B F 1$ have not been examined to determine whether similar epigenetic mutations will also arise, but we would predict that they could be isolated. Thus, the silencers, their associated binding proteins, and Sirlp likely comprise a structure whose role is to direct formation of heterochromatin and ensure its persistence but not to act as an integral component of that heterochromatin.

Temperature-sensitive alleles of $O R C 2$ and $O R C 5$ have been used to determine their requirement for silencing HMRa in cells blocked at M phase. In the context of a wild-type silencer no derepression of $H M R \mathbf{a}$ was seen when cells were shifted to the nonpermissive temperature. Slight derepression was observed, however, in backgrounds containing a compromised silencer, suggesting that ORC is required for maintenance of silencing at $M$ phase (Fox et al. 1995). Our results indicate that the $H M L$ E silencer and, by inference, ORC, is not required to maintain silencing at $H M L \alpha$ in $\mathrm{G}_{1}$ phase. Because our results indicate that silencers are required at some point in the cell cycle to promote inheritance, our results and those of Fox and colleagues (1995) are readily accommodated by the proposal that $M$ phase is at least one point within the cell cycle at which silencers are required. Using the FLP system, we have obtained pre- 
Holmes and Broach

Table 2. Strains used in this study

\begin{tabular}{|c|c|c|}
\hline Strain & Genotype & Source ${ }^{a}$ \\
\hline Y728 & HML $\alpha$ MATa HMRa ura3-52 leu2-3,112 ade2-1 lys1-1 his5-1 can1-100 & 1 \\
\hline Y729 & Y728, sir3::LEU2 & 1 \\
\hline Y799 & $\mathrm{Y} 728, H M L \alpha \mathrm{E} \Delta 79-113:: S U P 4 \mathrm{I} \Delta 242$ & 1 \\
\hline Y204a & Y799, leu2::LEU2-GAL10-FLP1 [cir $\left.{ }^{+}\right]$ & this study \\
\hline Y204b & Y799, leu2::LEU2-GAL10-FLP1 $\left[\mathrm{cir}^{\circ}\right]$ & this study \\
\hline Y2048 & $\mathrm{Y} 2047 \mathrm{~b}, \mathrm{E}(\Delta 79-113)-F R T-U R A 3-\mathrm{E}-F R T-H M L \alpha-\mathrm{I}\lrcorner 242$ & this study \\
\hline Y2049 & Y729, ura3::URA3-sir3-8 & this study \\
\hline Y1298 & Y728, leu2::LEU2-GAL10-MAT 2 & 2 \\
\hline
\end{tabular}

a(1) Mahoney and Broach (1989); (2) Mahoney et al. (1991).

liminary results that are consistent with this interpretation (S. Holmes and J.R. Broach, unpubl.). Thus, we conclude that ORC likely plays a role in inheritance of silencing but not directly in the heterochromatin structure.

\section{Silencers provide genomic memory}

Our results indicate that heterochromatin is not able to template its own reformation during each cell cycle; rather, left to its own devices, heterochromatin present at the beginning of a cell cycle is most often erased by the next cell cycle. Nonetheless, the transcription state of the silent cassettes can be inherited, as observed in strains bearing partial deletions of the silencer. Thus, because heterochromatin itself cannot ensure the persistence of transcriptional silencing at the silent cassettes from one generation to the next, the silencers themselves must provide the genomic memory of the expression state from preceding generations.

The existence of a genomic memory of silencing can be explained by the assumption that a silencer complex persists throughout the cell cycle. That is, the complex of silencer binding proteins-Rapl, Abf1, and/or ORCassembled on the silencer DNA would not only promote assembly of heterochromatin in the adjacent domain but also template its own duplication at each cell cycle. Accordingly, once assembled, this complex would be inherited efficiently and promote silencing in the progeny of silenced parents. Once disassembled, however, reformation would not readily occur; progeny would inherit an unassembled silencer and so would likely exhibit the same activated expression of the adjacent locus as did the parent cell. Consistent with the proposal that the silencer complex normally is never disassembled, Diffley and colleagues have shown that ORC remains bound to its site at $A R S 1$ throughout the cell cycle (Diffley and Cocker 1992; Diffley et al. 1994).

How might the silencer complex be maintained from one cell division to the next? One possibility is that the proteins that comprise the complex exhibit cooperative binding. In this case, the subset of silencer-binding proteins remaining associated with each of the sister chromosomes following DNA replication would seed the formation of a full silencer complex. A second, but not nec- essarily mutually exclusive, model would posit that the heterochromatin state induced by the silencer would positively influence the binding of the silencer proteins to the silencer. That is, silencer proteins may bind much tighter to silencer DNA packaged in the heterochromatin induced by the silencer proteins than to the same site packaged in active chromatin. In this case, the heterochromatin and the silencer complex are copropagated. Disassembly of either would lead to the dissolution of the other.

We have observed that in rare instances, the repressed state of the silent cassette can persist through an entire cell-cycle even in the absence of the silencer. One explanation for this phenomenon is that it occurs as the rare event in a stochastic process of chromatin segregation following DNA replication. If preexisting nucleosomes over the silenced domains segregate randomly following DNA replication (Sogo et al. 1986), then most often each sister strand would obtain a mixture of heterochromatic and newly deposited histones. If a preponderance of heterochromatic nucleosomes are required to effect transcriptional repression, then most of these new sister chromatids would not be repressed. In a few instances, however, one sister chromatid could inherit by chance a preponderance of heterochromatic nucleosomes, whereas the other could receive mostly newly deposited nucleosomes. In this case, the sister with the preponderance of heterochromatic nucleosome would yield a daughter cell with $H M L$ repressed. Obviously, as observed, this would not likely persist through a second generation.

\section{How does a silencer induce heterochromatin?}

Two models, summarized in Figure 8, could account for the role of silencers in promoting inheritance of the silenced state. In theory, the silencer could promote persistence of heterochromatin over the silenced loci from one generation to the next by keeping the heterochromatin from disassembling during the cell cycle. Alternatively, heterochromatin could spontaneously dissolve at some stage in the cell cycle, and the silencer could ensure its efficient reassembly during each cell cycle. Because no expression can be detected from the silent cassettes at any time during the cell cycle, this latter model 


\section{Silencer as:}

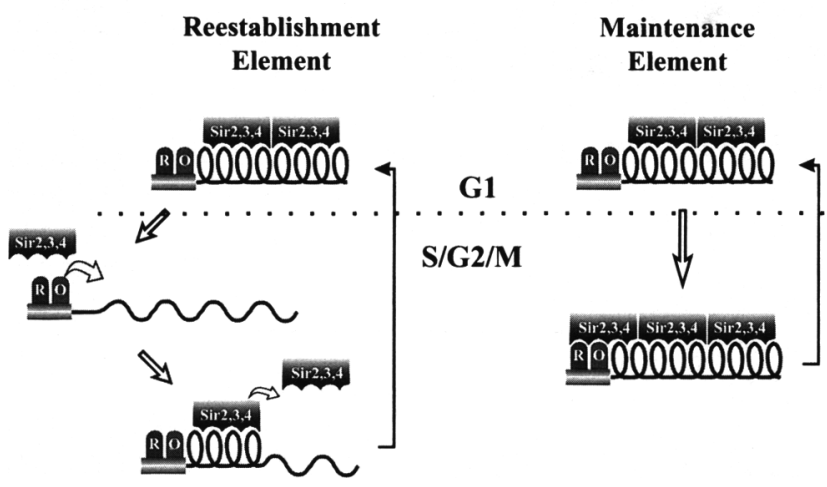

Figure 8. Possible mechanisms by which silencers promote inheritance of a heterochromatic domain. Silencers could act to reform heterochromatin after it is disrupted during progression through the cell cycle (diagramed at left). Reformation would have to occur immediately after disintegration, because no expression from HML is observed during any stage of the cell cycle. Alternatively, as diagramed on the right, silencers could stabilize the repressive structure to prevent dissolution of the heterochromatin during cell-cycle progression.

could be correct only if, during the period between disassembly and reassembly, the silent cassettes were inaccessible to the transcriptional machinery. Despite this constraint, two observations favor a disassembly-reassembly model. First, silenced promoters appear to be more accessible to challenge by specific transcription factors at a particular stage in the cell cycle-close to or during mitosis-than at other times (Aparicio and Gottschling 1994). Second, silenced domains that have been activated by dissolution of the heterochromatin over the region must pass through a specific stage in the cell cycle-S phase or later-to regain silencing (Miller and Nasymth 1984). Nonetheless, further information will be required to distinguish between these two models.

How does the silencer induce heterochromatin in adjacent domains? The function of silencers is mediated through the binding of specific factors--Raplp, Abflp, and ORC. These silencer-binding factors act to establish repression, at least in part, through recruitment of a complex of the Sir2, Sir3, and Sir4 proteins, which do not bind DNA themselves. For instance, Rapl protein shows genetic and physical interactions with the Sir $3 p$ and Sir4p proteins (Moretti et al. 1994). One member of the ORC complex, Orclp, has significant homology to Sir3p, suggesting that Sir3p may substitute for this protein in the silencer complex (S. Bell and B. Stillman, pers. comm.). Silencers, however, may serve additional roles besides simply recruiting SIR proteins. Chien et al. (1993) examined the ability of the SIR proteins to mediate silencing in the absence of silencer sequences by tethering them to the GAL4 DNA-binding domain and replacing the HMR silencers with the GAL4 UAS. They found no effect of tethering Sir $2 p$, Sir $3 p$, or Sir $4 p$ to
$H M R$, suggesting that localization of any one member of the $S I R$ complex is not sufficient to mediate repression. One additional function of the silencers may be to localize $H M L$ and $H M R$ to a specific subnuclear compartment. Rapl protein has been shown to localize to the nuclear periphery (Klein et al. 1992), whereas ARS sequences are preferentially bound to the nuclear scaffold in yeast (Amati and Gasser 1988). How these activities promote heterochromatin formation or transcriptional silencing remains unresolved.

\section{Silencing and silencers in other organisms-X chromosomes and Polycomb}

The observation in many systems of identical loci possessing distinct, heritable expression states implies that the loci are marked in a way that allows the expression state to be propagated from one cell division to the next. In many of these cases-such as X-chromosome inactivation-repression is effected by heterochromatin. Our results suggest that heterochromatin itself may not be sufficient to ensure maintenance of repression over successive cell divisions. Rather, we would anticipate that other features of each system provide the observed genomic memory. In Drosophila, the Polycomb group proteins and the attendant Polycomb response elements (PREs) serve to maintain the expression state of a number of developmentally regulated genes over successive cell divisions. In the absence of this complex, the repression state of loci such as Ultrabithorax $(U b x)$ can be established but rapidly decays (Pirotta 1995). This is somewhat different from the mode of action of the silencer complex in yeast, as maintenance functions are provided by a process quite distinct from that for establishment. Similarly, establishment and inheritance of X-chromosome inactivation are apparently accomplished by distinct processes. The XIC locus on the X-chromosome is required for establishment of repression and, accordingly, is formally similar to a yeast silencer. However, in contrast to the results we observe following deletion of the silencer, deletion of XIC after establishment of $\mathrm{X}$-chromosome inactivation does not appear to diminish inheritance of inactivation (Brown and Willard 1994). Thus, some other feature such as DNA methylation, which is clearly self-templating, may provide the mechanism of inheritance of silencing. Persistence of the repression through the cell division clearly presents a problem to all organisms; however, yeast, Drosophila and vertebrates appear to have solved this problem in distinct fashions.

\section{Materials and methods}

Strains and plasmids

All yeast strains used in this study are listed in Table 2 and were derived from strain Y728 [designated DMYl in Mahoney and Broach (1989)]. Strain Y2048 was constructed in several steps from Y799, which contains an unmarked deletion of the $H M L-I$ silencer and a substitution of the HML-E silencer with the SUP4 gene. Plasmid pFV17, a LEU2-marked, integrating vector con- 
taining the FLP gene controlled by the GAL10 promoter (Volkert and Broach 1986), was integrated into the leu2 locus in strain Y799 to yield strain Y2047a. Overexpression of the FLP gene in strains bearing the endogenous $2 \mu$ plasmid is toxic (Reynolds et al. 1987); accordingly, cells lacking the endogenous yeast $2 \mu$ plasmid were identified by plating strain Y2047a on galactose medium and recovering rare robust colonies. Several such clones were picked, tested by Southern analysis for the presence of the $2 \mu$ plasmid, and one that scored as plasmid-free was retained as strain Y2047b. Strain Y2047b was then made $\mathrm{Ura}^{+}$by transformation with plasmid pSH127 to introduce the first $F R T$ site. Plasmid pSH127 is derived from plasmid pDM11 and consists of a pUC19-URA3 plasmid carrying the $5.5-\mathrm{kb}$ BamHI-HindIII fragment spanning HMLa EJ79-113 with a FRT site inserted at the ClaI site adjacent to the E deletion. The FRT site consists of an 80-bp HindIII-SmaI fragment excised from plasmid pUC19-J3 (provided by M. Jayaram, University of Texas, Austin). One such transformant was then placed on 5-fluoro-orotic acid (5-FOA) plates to select for Ura isolates that had excised the SUP4 gene and most of pSH127, leaving the $F R T$ site adjacent to the E deletion at $H M L$. Such recombinants were identified by loss of SUP4 suppression of ade2-1 resulting in an $\mathrm{Ade}^{-}$phenotype. This strain was transformed to Ura. with integrating plasmid pSH128. Plasmid pSH128 was derived from plasmid pDM33 (Shei and Broach 1995) and consists of pUC19-URA3 carrying a 1.0-Kb Xbal fragment spanning the $\mathrm{E}$ site from $H M L$ into which a FRT site fragment has been inserted at the ClaI site. Integration of $\mathrm{pSH} 128$ restores the $H M L$-E silencer and places a second $F R T$ site telomere proximal to the $\mathrm{E}$ silencer (see Fig. 1).

Strain Y2049 was made by integration of plasmid pSH135 into strain Y729. Plasmid pSH135 is based on pRS406 /Sikorski and Hieter 1989) and contains the sir3-8 allele (Rine and Herskowitz 1987) rescued from strain Y408 by gap repair. Gap repair placed the temperature-sensitive mutation between the first BglII and unique NdeI sites of SIR3, within the open reading frame (Shore et al. 1984).

Strain Y1289 was described previously as strain GIY29 (Mahoney et al. 1991) and consists of strain Y728 into which a GAL10-MAT $\alpha 2$ construct has been integrated at leu2. The expression of $\alpha 2$ mRNA from galactose induced cells is not substantially higher than that seen in a normal MAT $\alpha$ strain.

\section{Media and growth of yeast cultures}

All cultures were grown in YP-raffinose medium $11 \%$ yeast extract, $2 \%$ Bacto-peptone, $2 \%$ raffinose). For galactose-induction experiments, galactose was added directly to cultures growing in YP-raffinose media to a final concentration of $2 \%$. $\alpha$-Factor was added to $\log$ phase cultures from a $2 \mathrm{mg} / \mathrm{ml}$ stock in methanol to a final concentration of $10 \mu \mathrm{g} / \mathrm{ml}$; to optimize sensitivity to $\alpha$-factor, the $\mathrm{pH}$ of the media was lowered by use of succinic acid $(1 \%)$ and sodium hydroxide $(0.075 \%)$.

\section{FLP assay}

Two criteria were used to assay the efficiency of the FLP-mediated deletion. Following galactose induction, cells were spread on glucose plates. Transfer to glucose represses transcription of the FLP gene and, because Flplp has a very short half-life in vivo (Volkert and Broach 1986), freezes the silencer in the configuration it was in at the time of plating. Any transiently maintained silencing was expected to decay in the 25-30 generations required to form a colony. The mating phenotype of the colony obtained from any plated cell thus reflected whether the plated cell had deleted or retained the silencer at the time of plating.
Cells lacking the silencer yielded nonmating colonies, whereas those with an intact silencer gave mating colonies. For each determination at least 200 colonies were assayed for mating. As an independent measure of the $F L P$-mediated deletion, Southern blots were performed on DNA isolated from cells collected at various times following induction. The efficiency of deletion was determined by quantitation of probed blots performed with a Molecular Dynamics PhosphorImager with ImageQuant software.

\section{$\alpha$-Factor sensitivity assay}

Sensitivity to mating pheromone was used as a monitor of $H M L$ expression. Cells were removed from cultures treated with $\alpha$-factor, and the fraction of unbudded cells was determined by microscopic examination. For each determination, at least 150 cells were assayed. The data shown are results from single experiments; each experiment presented is representative of at least three independent trials.

\section{Pedigree analysis}

Cultures were grown to $\log$ phase, treated with $\alpha$-factor until arrested, and induced with galactose for $1 \mathrm{hr}$. A small aliquot of cells was placed next to a streak of strain $17 \alpha$ on a YPD plate to confirm mating-factor arrest. Individual arrested cells were manipulated away from the $17 \alpha$ streak and observed periodically. When these cells budded, they were moved back into the presence of $\alpha$-factor. After cell division, the two cells were separated. Response to $\alpha$-factor was assayed $6-8 \mathrm{hr}$ later. For extended pedigrees, the process was repeated. To assay for the presence of the $H M L$-E silencer, individual cells were moved away from $\alpha$-factor and allowed to form colonies, and either mating tests or Southern analysis was performed.

\section{Acknowledgments}

We thank Miriam Braunstein and Steve West for helpful discussions, and Miriam Braunstein for critically reading this manuscript. This work was supported by National Institutes of Health (NIH) grant GM48540 to J.R.B. and an NIH postdoctoral fellowship to S.H.

The publication costs of this article were defrayed in part by payment of page charges. This article must therefore be hereby marked "advertisement" in accordance with 18 USC section 1734 solely to indicate this fact.

\section{Note added in proof}

A partial peptide sequence of the purified $150-\mathrm{kD}$ protein shown in Figure 6C has revealed significant homology with Drosophila $\mathrm{TAF}_{1 \mathrm{II}} 150$. Identity was found at 15 of 26 amino acid positions spanning four peptides. Moreover, of the 7 amino acids within these regions that are identical between the Drosophila $\mathrm{TAF}_{\mathrm{II}} 150$ and the yeast TSM-1 proteins, 6 were also identical in the human protein IJ. Kaufmann, C. Turck, and S.T. Smale, unpubl.).

\section{References}

Abraham, I., K.A. Nasmyth, J.N. Strathern, A.J.S. Klar, and J.B. Hicks. 1984. Regulation of mating-type information in yeast: Negative control requiring sequences both $5^{\prime}$ and $3^{\prime}$ to the regulated region. /. Mol. Biol. 176: 307-331.

Amati, B.B. and S.M. Gasser. 1988. Chromosomal ARS and CEN 
elements bind specifically to the yeast nuclear scaffold. Cell 54: 967-978.

Aparicio, O.M. and D.E. Gottschling. 1994. Overcoming telomeric silencing: A trans-activator competes to establish gene expression in a cell cycle-dependent way. Genes \& Dev. 8: $1133-1146$.

Bell, S.P., R. Kobayashi, and B. Stillman. 1993. Yeast origin recognition complex functions in transcription silencing and DNA replication. Science 262: 1844-1849.

Brand, A., L. Breeden, I. Abraham, R. Sternglanz, and K.A. Nasmyth. 1985. Characterization of a silencer in yeast: A DNA sequence with properties opposite to those of a transcriptional enhancer. Cell 41: 41-48.

Braunstein, M., A.B. Rose, S.G. Holmes, C.D. Allis, and J.R. Broach. 1993. Transcriptional silencing in yeast is associated with reduced nucleosome acetylation. Genes \& Dev. 7: 592 604.

Broach, J.R. and F.C. Volkert. 1991. Circular DNA plasmids of yeast. In The molecular biology of the yeast Saccharomyces: Genome dynamics, protein synthesis, and energetics/ed. J.R. Broach, E.W. Jones, and I.R. Pringlel, pp. 297-331. Cold Spring Harbor Laboratory Press, Cold Spring Harbor, NY.

Brown, C.J. and H.F. Willard. 1994. The human X-inactivation centre in not required for maintenance of $\mathrm{X}$-chromosome inactivation. Nature 368: 154-156.

Chien, C.T., S. Buck, R. Sternglanz, and D. Shore. 1993. Targeting of SIRI protein establishes transcriptional silencing at HM loci and telomeres in yeast. Cell 75: 531-54l.

Diffley, J.F. and J.H. Cocker. 1992. Protein-DNA interactions at a yeast replication origin. Nature 357: 169-172.

Diffley, J.F., J.H. Cocker, S.J. Dowell, and A. Rowley. 1994. Two steps in the assembly of complexes at yeast replication origins in vivo. Cell 78: 303-316.

Feldman, J.B., J.B. Hicks, and J.R. Broach. 1984. Identification of the sites required for repression of a silent mating type locus in yeast. J. Mol. Biol. 178: 815-834.

Fox, C.A., S. Loo, A. Dillin, and J. Rine. 1995. The origin recognition complex has essential functions in transcriptional silencing and chromosomal replication. Genes \& Dev. 9: $911-924$.

Haber, J.E. and J.R. George. 1979. A mutation that permits the expression of normally silent copies of mating-type information in Saccharomyces cerevisiae. Genetics 93: 13-35.

Hecht, A., T. Laroche, S. Strahl-Bolsinger, S.M. Gasser, and M. Grunstein. 1995. Histone H3 and H4 N-termini interact with SIR3 and SIR4 proteins: A molecular model for the formation of heterochromatin in yeast. Cell 80: 583-592.

Herskowitz, I., J. Rine, and J. Strathern. 1992. Mating-type determination and mating-type interconversion in Saccharomyces cerevisiae. In The molecular biology of the yeast Saccharomyces: Gene expression (ed. E.W. Jones, J.R. Pringle, and J.R. Broach), pp. 583-656. Cold Spring Harbor Laboratory Press, Cold Spring Harbor, NY.

Klar, A.J.S., S. Fogel, and K. MacLeod. 1979. MAR1-a regulator of HMa and HMa loci in Saccharomyces cerevisiae. Genetics 93: 37-50.

Klein, F., T. Laroche, M.E. Cardenas, J.F.-X. Hofman, D. Schweizer, and S.M. Gasser. 1992. Localization of RAPl and topoisomerase II in nuclei and meiotic chromosomes of yeast. J. Cell Biol. 117: 935-948.

Kurtz, S. and D. Shore. 1991. RAPl protein activates and silences transcription of mating-type genes in yeast. Genes \& Dev. 5: 616-628.

Laurenson, P. and J. Rine. 1992. Silencers, silencing, and heritable transcriptional states. Microbiol. Rev. 56: 543-560.

Loo, S. and J. Rine. 1994. Silencers and domains of generalized repression. Science 264: 1768-1771

Loo, S., C.A. Fox, J. Rine, R. Kobayashi, B. Stillman, and S. Bell. 1995a. The origin recognition complex in silencing, cell cycle progression, and DNA replication. Mol. Biol. Cell 6: 741756.

Loo, S., P. Laurenson, M. Foss, A. Dillin, and J. Rine. 1995b. Roles of $A B F 1, N P L 3$, and YCL54 in silencing in Saccharomyces cerevisiae. Genetics 141: 889-902.

Mahoney, D.J. and J.R. Broach. 1989. The HML mating-type cassette of Saccharomyces cerevisiae is regulated by two separate but functionally equivalent silencers. Mol. Cell. Biol. 9: 4621-4630.

Mahoney, D.J., R. Marquardt, G.J. Shei, A.B. Rose, and J.R. Broach. 1991. Mutations in the HML E silencer of Saccharomyces cerevisiae yield metastable inheritance of transcriptional repression. Genes \& Dev. 5: 605-615.

Marshall, M., S. Mahoney, A. Rose, J.B. Hicks, and J.R. Broach. 1987. Functional domains of SIR4, a gene required for position effect regulation in Saccharomyces cerevisiae. Mol. Cell. Biol. 7: 4441-4452.

Migeon, B. 1994. X-chromosome inactivation: Molecular mechanisms and genetic consequences. Trends Genet. 10: 230235

Miller, A. and K. Nasymth. 1984. Role of DNA replication in the repression of silent mating type loci in yeast. Nature 312: $247-251$.

Moretti, P., K. Freeman, L. Coodly, and D. Shore. 1994. Evidence that a complex of SIR proteins interacts with the silencer and telomere-binding protein RAPl. Genes \& Dev. 8: 2257-2269.

Palladino, F., T. Laroche, E. Gilson, A. Axelrod, L. Pillus, and S.M. Gasser. 1993. SIR3 and SIR4 proteins are required for the positioning and integrity of yeast telomeres. Cell 75: 543-555.

Pfeiffer, G.P., S.D. Steigerwald, R.S. Hansen, S.M. Gartler, and A.D. Riggs. 1990. Polymerase chain reaction-aided genomic sequencing of an X chromosome-lined CpG island: Methylation patterns suggest clonal inheritance, CpG site autonomy, and an explanation of activity state stability. Proc. Natl. Acad. Sci. 87: 8252-8256.

Pillus, L. and J. Rine. 1989. Epigenetic inheritance of transcription states in $S$. cerevisiae. Cell 59:637-647.

Pirotta, V. 1995. Chromatin complexes regulating gene expression in Drosophila. Curr. Opin. Genet. Dev. 5: 466-472.

Rastan, S. 1994. X chromosome inactivation and the Xist gene. Curr. Opin. Genet. Dev. 4: 292-297.

Reed, S.I. 1991. Pheromone signaling pathways in yeast. Curr. Opin. Genet. Dev. 1:391-396.

Reynolds, A.E., A.W. Murray, and J.W. Szostak. 1987. Roles of the $2 \mu \mathrm{m}$ gene products in stable maintenance of the $2 \mu \mathrm{m}$ plasmid of Saccharomyces cerevisiae. Mol. Cell. Biol. 7: 3566-3573.

Riggs, A.D. and G.P. Pfeiffer. 1992. X-chromosome inactivation and cell memory. Trends Genet. 8: 169-174.

Rine, J. and I. Herskowitz. 1987. Four genes responsible for a position effect on expression from $H M L$ and $H M R$ in Saccharomyces cerevisiae. Genetics 116: 9-22.

Rine, J.D., J.N. Strathern, J.B. Hicks, and I. Herskowitz. 1979. A suppressor of mating-type locus mutation in Saccharomyces cerevisiae: Evidence for and identification of cryptic mating type loci. Genetics 93: 877-901.

Shei, G.J. and J.R. Broach. 1995. Yeast silencers can act as orientation-dependent gene inactivation centers that respond to environmental signals. Mol. Cell. Biol. 15: 3496-3506.

Shore, D., M. Squire, and K. Nasmyth. 1984. Characterization of two genes required for the position-effect control of yeast 
mating-type genes. EMBO I. 3: 2817-2823.

Sikorski, R. and P. Hieter. 1989. A system of shuttle vectors and yeast host strains designed for efficient manipulation of DNA in Saccharomyces cerevisiae. Genetics 122: 19-27.

Singh, J. and A.J.S. Klar. 1992. Active genes in budding yeast display enhanced in vivo accessibility to foreign DNA methylases: A novel in vivo probe for chromatin structure of yeast. Genes \& Dev. 6: 186-196.

Sogo, J.M., H. Stahl, T. Koller, and R. Knippers. 1986. Structure of replicating simian virus 40 minichromosomes. The replication fork, core histone segregation and terminal structures. J. Mol. Biol. 189: 189-204.

Sussel, L., D. Vannier, and D. Shore. 1993. Epigenetic switching of transcriptional states: Cis- and trans-acting factors affecting establishment of silencing at the HMR locus in Saccharomyces cerevisiae. Mol. Cell. Biol. 13: 3919-3928.

Terleth, C., C.A. van Sluis, and P. van de Putte. 1989. Differential repair of UV damage in Saccharomyces cerevisiae. $\mathrm{Nu}$ cleic Acids Res. 17: 4433-4439.

Thompson, J.S., A. Hecht, and M. Grunstein. 1993. Histones and the regulation of heterochromatin in yeast. Cold Spring Harbor Symp. Quant. Biol. 58: 247-256.

Volkert, F.C. and J.R. Broach. 1986. Site-specific recombination promotes plasmid amplification in yeast. Cell 46: 541-550. 


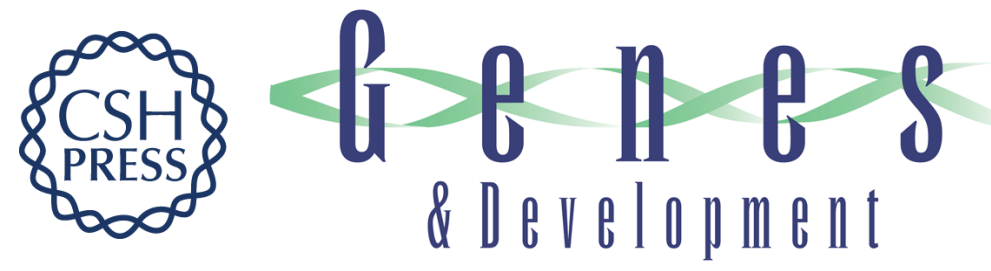

\section{Silencers are required for inheritance of the repressed state in yeast.}

S G Holmes and J R Broach

Genes Dev. 1996, 10:

Access the most recent version at doi:10.1101/gad.10.8.1021

$\begin{array}{ll}\text { References } & \begin{array}{l}\text { This article cites } 46 \text { articles, } 25 \text { of which can be accessed free at: } \\ \text { http://genesdev.cshlp.org/content/10/8/1021.full.html\#ref-list-1 }\end{array}\end{array}$

License

Email Alerting Receive free email alerts when new articles cite this article - sign up in the box at the top Service right corner of the article or click here.

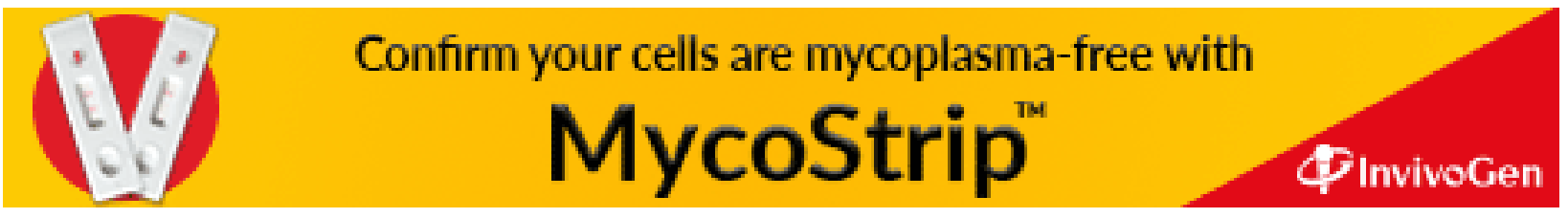

